\title{
Development of cross-resistance by Aspergillus fumigatus to clinical azoles following exposure to prochloraz, an agricultural azole
}

\author{
Isabel Faria-Ramos' ${ }^{1}$, Sofia Farinha', João Neves-Maia ${ }^{1}$, Pedro Ribeiro Tavares ${ }^{1}$, Isabel M Miranda ${ }^{1,2,3}$, \\ Letícia M Estevinho ${ }^{4}$, Cidália Pina-Vaz ${ }^{1,2,3,5}$ and Acácio G Rodrigues ${ }^{1,2,3,6^{*}}$
}

\begin{abstract}
Background: The purpose of this study was to unveil whether azole antifungals used in agriculture, similar to the clinical azoles used in humans, can evoke resistance among relevant human pathogens like Aspergillus fumigatus, an ubiquitous agent in nature. Additionally, cross-resistance with clinical azoles was investigated. Antifungal susceptibility testing of environmental and clinical isolates of A. fumigatus was performed according to the CLSI M38-A2 protocol. In vitro induction assays were conducted involving daily incubation of susceptible $A$. fumigatus isolates, at $35^{\circ} \mathrm{C}$ and 180 rpm, in fresh GYEP broth medium supplemented with Prochloraz (PCZ), a potent agricultural antifungal, for a period of 30 days. Minimal inhibitory concentrations (MIC) of PCZ and clinical azoles were monitored every ten days. In order to assess the stability of the developed MIC, the strains were afterwards sub-cultured for an additional 30 days in the absence of antifungal. Along the in vitro induction process, microscopic and macroscopic cultural observations were registered.
\end{abstract}

Results: MIC of PCZ increased 256 times after the initial exposure; cross-resistance to all tested clinical azoles was observed. The new MIC value of agricultural and of clinical azoles maintained stable in the absence of the selective PCZ pressure. PCZ exposure was also associated to morphological colony changes: macroscopically the colonies became mostly white, losing the typical pigmentation; microscopic examination revealed the absence of conidiation.

Conclusions: PCZ exposure induced Aspergillus fumigatus morphological changes and an evident increase of MIC value to PCZ as well as the development of cross-resistance with posaconazole, itraconazole and voriconazole.

Keywords: Aspergillus fumigatus, Cross-resistance, Clinical and agricultural azoles

\section{Background}

The ubiquitous saprophytic mould Aspergillus fumigatus is known to cause a spectrum of diseases in humans, including allergic syndromes, noninvasive infections, and invasive aspergillosis, a condition associated with significant morbidity and mortality [1]. A. fumigatus is one of the human pathogenic fungi that have a natural habitat in the environment, including soil and plants [2]. Some members of the azole drug class, which includes voriconazole (VRC) and posaconazole (POS), have been shown

\footnotetext{
*Correspondence: agr@med.up.pt

'Microbiology Department, Faculty of Medicine, University of Porto, 4200-319 Porto, Portugal

${ }^{2}$ Cardiovascular Research \& Development Unit, Faculty of Medicine, University of Porto, Porto, Portugal

Full list of author information is available at the end of the article
}

to be effective in the treatment of invasive aspergillosis [3], and for a long time, azole resistance among clinical A. fumigatus isolates was considered to be an uncommon finding. However, multiazole resistance is emerging and is increasingly recognized as a cause of treatment failure $[4,5]$. In agriculture, thousands of tons of azoles are sold annually for the purpose of plant protection, either to prevent or to control fungal growth that can cause extensive loss of crops or to ease the problem of postharvest spoilage of plants and fruits [6]. The mechanism of action of all azoles - irrespectively of their chemical structure and variable biological properties - is based on its interference with the activity of fungal lanosterol 14 alpha-demethylase, an enzyme encoded by Cyp51A gene in A. fumigatus that is responsible for the transformation 
of lanosterol in ergosterol, an essential component of the fungal cytoplasmatic membrane. The inhibition of ergosterol formation results in cell membrane disorganization and impairment of fungal growth. Therefore, azoles are considered fungistatic rather than fungicidal, and it is well known that a strong and persistent antimicrobial pressure can lead to the selection of resistant clones, particularly if the drug effect is static rather than microbicidal [7]. Since azoles are the mainstay treatment for both human and agricultural fungal diseases, a major concern is the predictable emergence of cross-resistance to clinical $A$. fumigatus isolates that is already observed in several countries, driven by the massive use of azole fungicides in agriculture, which have the same mechanism of action as those used in humans [7-11]. The aim of our study was to investigate whether Prochloraz (PCZ), an azole extensively used in agriculture, could be associated with the development of cross-resistance to clinical azoles among A. fumigatus.

\section{Results and discussion}

The three isolates developed a progressive increment of PCZ minimal inhibitory concentrations (MIC) value. In addition, a concomitant increase of the MIC of VRC, POS and Itraconazole (ITZ) was also observed (Table 1). During the induction assay, MIC of PCZ increased 256 times from day 0 until day 30. Concerning the clinical azoles, cross-resistance was developed since all isolates changed from a susceptible to a resistant phenotype, according to Meletiadis and colleagues [12].

Table 1 Susceptibility pattern of tested A. fumigatus isolates to Prochloraz and clinical azoles

\begin{tabular}{|c|c|c|c|c|c|c|}
\hline \multirow[t]{2}{*}{$\begin{array}{c}A . \\
\text { fumigatus } \\
\text { isolate }\end{array}$} & \multirow[t]{2}{*}{$\begin{array}{l}\text { Time of } \\
\text { exposure } \\
\text { (days) }\end{array}$} & \multicolumn{5}{|c|}{$\mathrm{MIC}(\mathrm{mg} / \mathrm{L})$} \\
\hline & & $\mathrm{PCZ}$ & VRC & POS & ITZ & FLC \\
\hline \multirow[t]{5}{*}{ LMF05 } & 0 & 0.125 & 0.125 & 0.25 & 2 & $>64$ \\
\hline & 10 & 0.25 & 0.25 & 0.5 & 2 & $>64$ \\
\hline & 20 & 8 & 2 & 1 & 4 & $>64$ \\
\hline & 30 & 32 & 8 & 2 & 8 & $>64$ \\
\hline & $\varnothing 30$ & 32 & 2 & 2 & 2 & $>64$ \\
\hline \multirow[t]{5}{*}{ LMF11 } & 0 & 0.125 & 0.25 & 0.125 & 0.5 & $>64$ \\
\hline & 10 & 0.125 & 2 & 0.25 & 1 & $>64$ \\
\hline & 20 & 8 & 8 & 1 & 2 & $>64$ \\
\hline & 30 & 32 & $>16$ & 4 & 4 & $>64$ \\
\hline & $\varnothing 30$ & 32 & 2 & 1 & 0.5 & $>64$ \\
\hline \multirow[t]{5}{*}{ LMN60 } & 0 & 0.25 & 0.25 & 0.125 & 0.25 & $>64$ \\
\hline & 10 & 4 & 8 & 0.25 & 1 & $>64$ \\
\hline & 20 & 8 & 8 & 0.5 & 2 & $>64$ \\
\hline & 30 & 64 & $>16$ & 4 & 4 & $>64$ \\
\hline & $\varnothing 30$ & 64 & 2 & 1 & 0.25 & $>64$ \\
\hline
\end{tabular}

PCZ, Prochloraz; VRC, Voriconazole; POS, Posaconazole; ITZ, Itraconazole; FLC, Fluconazole; $\varnothing$, MIC after 30 days of culture in the absence of PCZ.
There are several studies that have characterized azole resistance in $A$. fumigatus, and most recently some addressed the possible cross-resistance between environmental and medical azoles [8-11]. Our study demonstrated the time frame between the introduction of a widely used agricultural antifungal and the emergence of cross-resistance to medical triazoles. During the induction assay, we found that besides the emergence of cross-resistance, PCZ exposure caused marked morphological colony changes, both macroscopically and microscopically. Macroscopic modification of the pigmentation of $A$. fumigatus colonies, changing from the original green colour to white (Figure 1A, B and $C$ ) was remarkable at the beginning of the assay. With the increase of MIC values of PCZ the colonies became scarcer, smaller and totally white (Figure 1C). Microscopic examination showed a progressive absence of conidiation: the original strain (Figure 1A) showed normal microscopic features regarding conidiation (Figure 2A) while almost white colonies (Figure 1B) showed nearly complete absence of conidiation (Figure 2B). The totally white mycelia (Figure 1C) corresponded solely to hyphae and immature little conidiophore structures without conidia (Figure 2C). These changes in pigmentation and in conidiation as a consequence of exposure to azoles have already been reported. Varanasi and colleagues speculate that azoles may bind to a phytochrome-like regulatory molecule inhibiting the initiation and subsequent development of conidiophores in Aspergillus species [13]. Such mechanism of action could also explain the different levels of inhibition displayed by other tested azoles and why echinocandins and polyenes did not show this effect [13]. Notably, such morphological changes may be responsible for laboratorial diagnostic misidentification of the fungal genus/species [14]. The high MIC values for PCZ that were achieved in vitro maintained stable following removal of the selective pressure of the drug. For VRC, the MIC value decreased only after 30 days of incubation without the selective pressure, changing the susceptibility phenotype from resistant to intermediate. For POS, the developed MIC value also decreased but not enough to change the phenotype of resistance. Regarding ITZ, for both LMF11 and LMN60, it was observed the complete reversibility of the resistant phenotype in the absence of PCZ, ie, the MIC reverted to the initial value (susceptible). However, strain LMF05 had, since day zero, ITZ MIC of $2 \mathrm{mg} / \mathrm{L}$, which falls in resistant category. In all the isolates conidiation reappeared together with the typical green colour of mature colonies following the removal of PCZ.

Since PCZ was responsible for the emergence of stable resistance to itself and to very important medical triazoles in A. fumigatus, a resistance mechanism may have been developed. Previous reports describe cyp51A mutation, efflux pump overexpression and/or target upregulation as the main mechanisms responsible for 


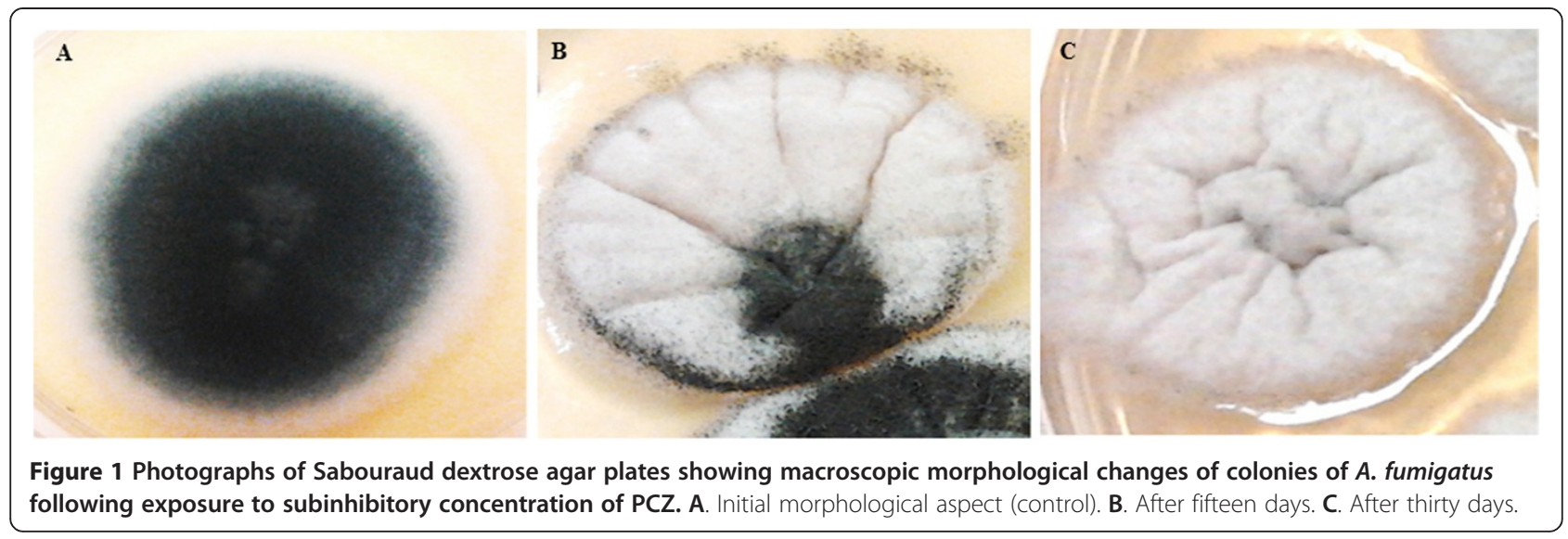

such resistance [15-17]. A clonal expansion of isolates harbouring the TR34/L98H mutation has been reported across several countries [15-18]. Interestingly, besides the fact that these resistant isolates are less genetically variable than susceptible ones, no impact on fitness was observed [18]. The phenotypic results (Figures 1 and 2) and the stability of the developed resistance (Table 1) herein reported suggest the same. Future studies aiming to assess the underlying molecular resistance mechanisms, not only from these induced resistant strains but also from isolates with naturally high MIC values to PCZ and resistant to medical azoles without previous in vitro induction, will certainly be our next step. Meanwhile, our study suggests that the abuse of azole antifungals in nature may cause serious human health problems since azoleresistance and cross-resistance has the potential to further compromise the efficacy of clinical azoles in the future $[4,17-20]$. Furthermore, we can speculate that the exposure of clinically relevant moulds other than A. fumigatus to agricultural azoles may also be associated with the emergence of cross-resistance to clinical azoles. Several compounds are being tested in order to find new antifungal alternatives, anticipating the possible loss of efficacy of clinical azoles [21]. On the other hand, efforts should be made to find safer compounds to use in agriculture.

\section{Conclusions}

In order to assess the real dimension of Aspergillus resistance, a susceptibility test should be performed in all isolates from patients with Aspergillus infection. Moreover, for patients with severe infection initial combination therapy may be considered in geographical areas with high prevalence of environmental azole resistant isolates. Ultimately, surveillance studies in both clinical and in environment settings should be conducted in order to provide updated local data regarding susceptibility profiles.

\section{Methods}

\section{Organisms}

Two clinical isolates of A. fumigatus, LMF05 and LMF11, and one environmental $A$. fumigatus isolate (LMN60, recovered from a garden nearby the hospital), were used in this study. The isolates were identified as belonging to $A$.

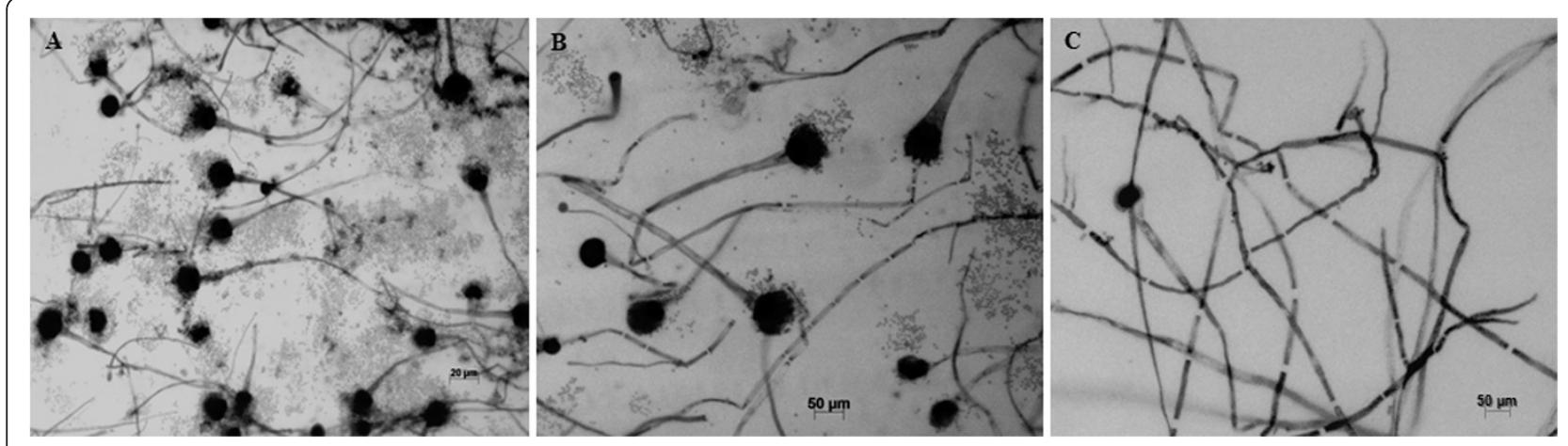

Figure 2 Photomicrographs of Aspergillus fumigatus colonies using the cellotape flag technique preparation with lactophenol cotton blue staining. Microscopic morphological changes in the development of conidiation of A. fumigatus following exposure to subinhibitory concentration of PCZ. A. Initial morphological aspect (control). B. After fifteen days. C. After thirty days. 
fumigatus species by macroscopic and microscopic morphology, the ability to grow at $48^{\circ} \mathrm{C}$ and by using MALDI-TOF MS to accurately discriminate $A$. fumigatus from a new sibling species $A$. lentulus, which cannot be distinguished by morphological characteristics or growth peculiarities [22]. Long-term preservation of conidial suspensions of the isolates was made in a GYEP medium ( $2 \%$ glucose, $0.3 \%$ yeast extract, $1 \%$ peptone) broth supplemented with $10 \%$ glycerol and stored at $-80^{\circ} \mathrm{C}$. Working cultures were subsequently maintained during 2 weeks on Sabouraud dextrose agar slants and plates at $4^{\circ} \mathrm{C}$.

\section{Antifungal agents and susceptibility profile}

PCZ is an imidazole and one of the main drugs used within European Union for crop protection [23]. This ergosterol biosynthesis inhibitor was selected as a representative of agricultural azoles after a previous MIC screening, where it showed to be the less active agricultural drug on the selected strains, ie, it had the lower MIC values, which was a prerequisite for this induction experiment. Fluconazole (FLC), VRC, POS and ITZ were selected as clinical azoles. PCZ was ressuspended in $80 \%$ acetone solution at a final concentration of $5 \mathrm{mg} / \mathrm{L}$. Clinical azoles were dissolved in dimethysulphoxide (DMSO) to obtain stock solutions of $10 \mathrm{mg} / \mathrm{L}$. All drugs were stored at $-20^{\circ} \mathrm{C}$. Broth microdilution susceptibility assay was performed according to the Clinical and Laboratory Standards Institute M38-A2 protocol in order to evaluate the initial MIC of PCZ and of all the clinical azoles [24]. Drug concentration ranged from 0.125 to $64 \mathrm{mg} / \mathrm{L}$ of FLC and PCZ; and 0.0313 to $16 \mathrm{mg} / \mathrm{L}$ of POS, VRC and ITZ. A. fumigatus ATCC 46645 was included for quality control of susceptibility testing. Also, FLC was used as control, since $A$. fumigatus shows a non-susceptible phenotype and MIC is most often above $64 \mathrm{mg} / \mathrm{L}$ for this species. MIC of azoles was defined as the lowest concentration of the drug that produced no visible growth following 48 hours of incubation. MIC determination was repeated at least twice.

\section{In vitro induction experiments}

Induction experiments were performed with the agricultural azole PCZ. A. fumigatus isolates were grown on Saboraud dextrose agar at $35^{\circ} \mathrm{C}$ for $72 \mathrm{~h}$; conidia were harvested by flooding the surface of the slants with phosphate-buffered saline (PBS) containing 0.025\% (vol/ vol) tween 80 while gently rocking. The conidial suspensions were then adjusted using specific spectrophotometric readings at $550 \mathrm{~nm}$ to a final concentration of $5 \times 10^{4}$ conidia per militer [25]; one militer of each distinct isolate suspension was transferred to $9 \mathrm{ml}$ of GYEP broth supplemented with sub-inhibitory concentrations of PCZ $(0.06 \mathrm{mg} / \mathrm{L}$ for both LMF05 and LMF11; $0.125 \mathrm{mg} / \mathrm{L}$ for LMN60) and incubated overnight at $35^{\circ} \mathrm{C}$ with agitation (180 rpm). Daily, after vigorous vortexing for 60 seconds, one militer from each culture was transferred to fresh GYEP medium supplemented with PCZ and in parallel, $1 \mathrm{ml}$ of culture was added with $10 \%$ glycerol and frozen at $-80^{\circ} \mathrm{C}$. This procedure was repeated along thirty consecutive days.

\section{Susceptibility testing/ Stability of in vitro developed resistance phenotype}

MICs of PCZ were determined every ten days along the thirty days of induction assay. No official breakpoints are yet defined for PCZ; therefore, whenever a marked MIC increase was observed (four fold the initial PCZ MIC), the MIC values of clinical antifungals were determined.

In order to assess the stability of the developed MIC increment to PCZ and of the developed cross-resistance to clinical azoles, the induced strains were afterwards sub-cultured for an additional thirty days in the absence of the drug and MIC values re-determined, as previously described.

\section{Culture macro and micro morphology}

Along the induction process, every two days, a loopful was inoculated in Saboraud Agar slants to check for viability and purity of culture. Macro and microscopical growth characteristics were registered. Colony morphology and pigmentation were recorded photographically using a Reflex Nikon D3200 Camera and images were processed by Adobe Photo Deluxe Image Processing Program. Microscopic images of hyphae changes from the original A. fumigatus strain and from the resistant induced strain were captured with a Zeiss-Axioplan-2 microscope equipped with Axio Cam. AxioVision 3.0 digital imaging software was used for editing.

\section{Competing interests}

The authors declare that they have no competing interests.

\section{Authors' contributions}

IFR, IMM and AGR: conceived the study and designed the experiments; IFR and SF: performed the experiments; IFR, SF, JNM and PRT: analysed the data; IMM, LME, CPV and AGR: Contributed with reagents/material and analysis tools; IFR, SF, IMM and AGR: wrote and revised the manuscript. All authors read and approved the final manuscript.

\section{Acknowledgments}

IFR and IMM are supported by FCT (Fundação Ciência e Tecnologia). IFR is supported by FCT PhD grant (SFRH/BD/91155/2012). I.MM is supported by FCT, Ciência 2008 and co-financed by the European Social Fund. The authors would like to thank Isabel Santos for the excellent technical assistance. Part of the results was presented at the $52^{\text {nd }}$ Interscience Conference on Antimicrobial Agents and Chemotherapy, ICAAC, held San Francisco, USA, September 2012.

\section{Author details}

${ }^{1}$ Microbiology Department, Faculty of Medicine, University of Porto, 4200-319 Porto, Portugal. ${ }^{2}$ Cardiovascular Research \& Development Unit, Faculty of Medicine, University of Porto, Porto, Portugal. ${ }^{3}$ CINTESIS - Center for Health Technology and Services Research, Faculty of Medicine of the University of 
Porto, Porto, Portugal. ${ }^{4}$ Department of Biology and Biotechnology, CIMO-Mountain Research Center, Agricultural College of Bragança, Polytechnic Institute of Bragança, Porto, Portugal. ${ }^{5}$ Microbiology Department, Centro Hospitalar de São João, Porto, Portugal. ${ }^{6}$ Burn Unit, Centro Hospitalar de São João, Porto, Portugal.

Received: 6 March 2014 Accepted: 4 June 2014

Published: 11 June 2014

\section{References}

1. Denning DW, Riniotis K, Dobrashian R, Sambatakou H: Chronic cavitary and fibrosing pulmonary and pleural aspergillosis: case series, proposed nomenclature change, and review. Clin Infect Dis 2003, 37:S265-S280.

2. Shapiro RS, Robbins N, Cowen LE: Regulatory circuitry governing fungal development, drug resistance, and disease. Microbiol Mol Biol Rev 2011, 75:213-267.

3. Walsh TJ, Anaissie EJ, Denning DW, Herbrecht R, Kontoyiannis DP, Marr KA, Morrison VA, Segal BH, Steinbach WJ, Stevens DA, van Burik J, Wingard JR, Patterson TF: Treatment of Aspergillosis: Clinical Practice Guidelines of the Infectious Diseases Society of America. Clin Infect Dis 2008, 46:327-360

4. Howard SJ, Cerar D, Anderson MJ, Albarrag A, Fisher MC, Pasqualotto AC, Laverdiere M, Arendrup MC, Perlin DS, Denning DW: Frequency and evolution of Azole resistance in Aspergillus fumigatus associated with treatment failure. Emerg Infect Dis 2009, 15:1068-1076.

5. Arikan-Akdagli S: Azole resistance in Aspergillus: global status in Europe and Asia. Ann N Y Acad Sci 2012, 1272:9-14.

6. Hof H: Critical annotations to the use of azole antifungals for plant protection. Antimicrob Agents Chemother 2001, 45:2897-2990

7. Bowyer P, Denning DW: Environmental fungicides and triazole resistance in Aspergillus. Pest Manag Sci 2014, 70:173-178.

8. Snelders E, Camps SM, Karawajczyk A, Schaftenaar G, Kema GH, van der Lee $\mathrm{HA}$, Klaassen $\mathrm{CH}$, Melchers WJ, Verweij PE: Triazole fungicides can induce cross-resistance tomedical triazoles in Aspergillus fumigatus. PLoS One 2012, 7:e31801.

9. Snelders E, Veld RA HI't, Rijs AJ, Kema GH, Melchers WJ, Verweij PE: Possible Environmental Origin of Resistance of Aspergillus fumigatus to Medical Triazoles. Appl Environ Microbiol 2009, 75:4053-4057.

10. Verweij PE, Snelders E, Kema GH, Mellado E, Melchers WJ: Azole resistance in Aspergillus fumigatus: a side-effect of environmental fungicide use? Lancet Infect Dis 2009, 9:789-795.

11. Stensvold CR, Jorgensen LN, Arendrup MC: Azole-Resistant Invasive Aspergillosis: Relationship to Agriculture. Curr Fungal Infect Rep 2012, 6:178-191.

12. Meletiadis J, Mavridou E, Melchers WJ, Mouton JW, Verweij PE: Epidemiological cutoff values for azoles and Aspergillus fumigatus based on a novel mathematical approach incorporating cyp51A sequence analysis. Antimicrob Agents Chemother 2012, 56:2524-2529.

13. Varanasi NL, Baskaran I, Alangaden GJ, Chandrasekar PH, Manavathu EK: Novel effect of voriconazole on conidiation of Aspergillus species. Int $J$ Antimicrob Agents 2004, 23:72-79.

14. Sangoi AR, Rogers WM, Longacre TA, Montoya JG, Baron EJ, Banaei N: Challenges and pitfalls of morphologic identification of fungal infections in histologic and cytologic specimens: a ten-year retrospective review at a single institution. Am J Clin Pathol 2009, 131:364-375.

15. Verweij PE, Kema GH, Zwaan B, Melchers WJ: Triazole fungicides and the selection of resistance to medical triazoles in the opportunistic mould Aspergillus fumigatus. Pest Manag Sci 2013, 69:165-170.

16. Fraczek MG, Bromley M, Buied A, Moore CB, Rajendran R, Rautemaa $R$, Ramage G, Denning DW, Bowyer P: The cdr1B efflux transporter is associated with non-cyp51a-mediated itraconazole resistance in Aspergillus fumigatus. J Antimicrob Chemother 2013, 68:1486-1496.

17. Vermeulen E, Lagrou K, Verweij PE: Azole resistance in Aspergillus fumigatus: a growing public health concern. Curr Opin Infect Dis 2013, 26:493-500.

18. Chowdhary A, Kathuria S, Xu J, Meis JF: Emergence of Azole- Resistant Aspergillus fumigatus Strains due to Agricultural Azole Use Creates an Increasing Threat to Human Health. PLoS Pathog 2013, 9:1003633.

19. Gisi U: Assessment of selection and resistance risk for DMI fungicides in Aspergillus fumigatus in agriculture and medicine: A critical review. Pest Manag Sci 2014, 70(3):352-364.
20. Hof $\mathrm{H}$ : Is there a serious risk of resistance development to azoles among fungi due to the widespread use and long-term application of azole antifungals in medicine? Drug Resist Updat 2008, 11:25-31.

21. Geronikaki A, Fesatidou M, Kartsev V, Macaev F: Synthesis and biological evaluation of potent antifungal agents. Curr Top Med Chem 2013, 13:2684-2733

22. Verwer PE, van Leeuwen WB, Girard V, Monnin V, van Belkum A, Staab JF, Verbrugh HA, Bakker-Woudenberg IA, van de Sande WW: Discrimination of Aspergillus lentulus from Aspergillus fumigatus by Raman spectroscopy and MALDI-TOF MS. Eur J Clin Microbiol Infect Dis 2014, 33:245-251.

23. European Comission: The use of plant protection products in the European Union. 2007 [http://epp.eurostat.ec.europa.eu/portal/page/portal/ product_details/publication?p_product_code=KS-76-06-669]

24. Clinical and Laboratory Standards Institute: Reference Method for Broth Dilution Antifungal Susceptibility Testing of Filamentous Fungi; Approved Standard- Second Edition. Wayne, PA, USA: CLSI M38-A2; 2002.

25. Araujo R, Rodrigues AG, Pina-Vaz C: A fast, practical and reproducible procedure for the standardization of the cell density of an Aspergillus suspension. J Med Microbiol 2004, 53:783-786.

doi:10.1186/1471-2180-14-155

Cite this article as: Faria-Ramos et al:: Development of cross-resistance by Aspergillus fumigatus to clinical azoles following exposure to prochloraz, an agricultural azole. BMC Microbiology 2014 14:155.

\section{Submit your next manuscript to BioMed Central and take full advantage of:}

- Convenient online submission

- Thorough peer review

- No space constraints or color figure charges

- Immediate publication on acceptance

- Inclusion in PubMed, CAS, Scopus and Google Scholar

- Research which is freely available for redistribution 